\title{
Transcription from a TATA-less promoter requires a multisubunit TFIID complex
}

\author{
B. Franklin Pugh' ${ }^{1}$ and Robert Tiian \\ Howard Hughes Medical Institute, Department of Molecular and Cell Biology, University of California, Berkeley, \\ California 94720 USA
}

In eukaryotes, the TATA box-binding protein (TBP) is responsible for nucleating assembly of the transcription initiation machinery. Here, we report that a TFIID complex containing TBP is essential for transcription even at a promoter that lacks a TATA box. Immunopurification of TFIID reveals that the active species in reconstituting TATA-less transcription is a multisubunit complex consisting of TBP and many TBP-associated factors (TAFs).

[Key Words: TATA box-binding protein; transcription; TFIID complex; TATA-independent; TBP]

Received August 20, 1991; revised version accepted September 13, 1991.

The promoters of eukaryotic protein-coding genes differ widely but can be generally classified as containing or lacking a TATA box (consensus TATAAAA), which is typically located 25-30 nucleotides upstream from the transcriptional start site (or initiator element). The TATA box binds TFIID and, along with the initiator, helps direct RNA polymerase II and other required basal initiation factors (TFIIA, TFIIB, TFIIE, TFIIF, and TFIIG) to assemble efficiently into a stable preinitiation complex (Saltzman and Weinmann 1989).

Recently, cDNA clones of the TATA box-binding protein (TBP) from yeast, mammals, flies, and plants have been isolated, and protein sequence comparisons reveal a striking bipartite structure /Cavallini et al. 1989; Eisenmann et al. 1989; Hahn et al. 1989a; Horikoshi et al. 1989; Schmidt et al. 1989; Fikes et al. 1990; Gasch et al. 1990; Hoey et al. 1990; Hoffmann et al. 1990a,b; Kao et al. 1990; Muhich et al. 1990; Peterson et al. 1990). The carboxy-terminal 180 residues are rich in basic amino acids, contain a tandem repeat, and are $>80 \%$ identical in amino acid sequence among the different organisms. This highly conserved carboxy-terminal domain is necessary and sufficient for the TBP to bind the TATA box and recruit TFIIA and TFIIB to the promoter (Buratowski et al. 1989; Hoey et al. 1990; Horikoshi et al. 1990; Peterson et al. 1990). Most significantly, this region also suffices to replace the endogenous human TFIID fraction in supporting a basal unregulated level of transcription initiation (Hoey et al. 1990; Horikoshi et al. 1990; Peterson et al. 1990). In contrast, the divergent amino-terminal portion of the various TBPs shares very little sequence sim-

${ }^{1}$ Present address: Department of Molecular and Cell Biology, The Pennsylvania State University, University Park, Pennsylvania 16802 USA. ilarity and, in some cases, is rich in glutamine residues. Although the function of the amino-terminal domain is poorly characterized, in higher eukaryotes this speciesspecific domain appears to indirectly mediate promoter regulation by sequence-specific transcriptional activators such as Spl (Peterson et al. 1990; Pugh and Tjian 1990).

Biochemical characterization of the recombinant human TBP overexpressed in HeLa cells reveals that it exists as a $38-\mathrm{kD}$ monomer in solution (Peterson et al. 1990). In contrast, its natural or endogenous counterpart behaves as a much larger macromolecular complex during gel filtration chromatography and glycerol gradient sedimentation (Pugh and Tiian 1990; Dynlacht et al. 1991; N. Tanese and R. Tjian, unpubl.). Throughout this paper we will refer to the $38-\mathrm{kD}$ monomer as the human TATA box-binding protein (hTBP) and to the high-molecular-weight endogenous protein as the TFIID complex. Previous findings suggest that TBP alone is not sufficient to carry out all of the activities generally attributed to the partially purified fraction containing the TFIID complex, especially transcriptional regulation by upstream activators (Hoffmann et al. 1990b; Kambadur et al. 1990; Peterson et al. 1990; Pugh and Tjian 1990; Dynlacht et al. 1991; Tanese and Tjian 1991; Workman et al. 1991). To account for this difference, transcriptional activators such as Spl and CCAAT box transcription factor $(\mathrm{CTF})$ were proposed to regulate transcription through coactivators or adaptors that copurified with the TFIID complex (Pugh and Tjian 1990).

If TBP is critical for transcription and is recruited to the promoter by the TATA box, how can some promoters function in the absence of a TATA box? Is TBP or the TFIID complex required at such promoters? If so, how does it recognize the promoter? These so-called TATA- 
less promoters regulate a large variety of cellular and viral genes, including those coding for dihydrofolate reductase, c-H-ras, adenine deaminase, TGF- $\alpha$, thymidylate synthetase, and SV40 late gene products (Dynan 1986; Swick et al. 1989 and refs. therein). One common and intriguing property of many TATA-less promoters is the presence of multiple GC boxes /consensus GGGGCGGGGC) that bind the transcriptional activator Spl. Transcription initiation directed by these templates is critically dependent on $\mathrm{Sp} 1$; therefore, understanding how $\mathrm{Spl}$ activates transcription at such TATA-less promoters might provide new insights into the mechanism of initiation.

In an effort to understand how Spl can activate a TATA-less promoter, we previously addressed the factor requirement at a promoter containing only Spl-binding sites and an initiator element. Spl was found to utilize the same fractionated basal initiation factors, including the partially purified TFIID fraction, that are required for transcription at TATA-containing promoters (Pugh and Tjian 1990). In addition, a novel activity was described that was present in the HeLa TFIID fraction but distinct from TBP and the Spl coactivator and was required only for activation of a TATA-less promoter by Spl (Pugh and Tjian 1990). In the presence of promoter-bound Sp1, this so-called tethering factor apparently could function as a substitute for the TATA box in transcription assays in vitro. These observations led to the proposal that Spl plays an essential role in assembling the basal initiation factors, possibly by anchoring TBP to the TATA-less template via the tethering factor. Our hypothesis is that TFIID is a complex of distinct transcription factors, and TBP is the core subunit that is required for transcription even at a TATA-less template. However, neither of these assumptions has been experimentally established.

In this paper we address whether the proposed TFIID complex plays an important role in transcription at a TATA-less promoter. Affinity-purified antibodies directed against the recombinant hTBP were used to specifically inhibit and/or remove from the HeLa nuclear extract the endogenous TBP, along with any factors that might be tightly associated with it, such as the tethering factor. The composition of the immunoprecipitated TFIID complex was determined by gel electrophoresis, and the depleted extracts were then tested for their ability to transcribe an Sp1-activated TATA-less promoter in the presence or absence of added purified recombinant TBP or purified immunoprecipitated TFIID complex. The results suggest similar requirements for the TFIID complex at promoters containing or lacking the TATA box but distinct mechanisms of transcriptional regulation by $\mathrm{Sp} 1$.

\section{Results}

Properties of a TATA-less promoter

Before addressing whether the TBP or the proposed TFIID complex is required at a TATA-less promoter, it is first necessary to establish that the template being used is behaving as a TATA-less promoter. The lack of a ca- nonical TATA-like sequence 30 nucleotides upstream of the transcriptional start site may not be sufficient criteria to define a TATA-less promoter. For example, the adenovirus EIVa2 promoter has been described as a TATA-less promoter (Carcamo et al. 1989). However, a recent study identified a TATA box located at position +15 , which is required for specific and efficient initiation from the +1 site of the promoter /Carcamo et al. 1990). In addition, TBP and factors in the TFIID fraction have the potential to recognize the promoter through a number of sequences unrelated to the TATAAAA motif (Hahn et al. 1989b; Singer et al. 1990). A functionally TATA-less promoter should have no intrinsic ability to specifically bind TBP or the TFIID complex directly and thus direct virtually no basal level transcription in the absence of a promoter-bound activator such as Spl.

The template chosen for this study, $\mathrm{G}_{6} \mathrm{I}$, consists of multiple Spl-binding sites upstream of an initiator element (Smale and Baltimore 1989; Pugh and Tjian 1990). No sequence upstream or within 30 nucleotides downstream of the transcriptional start site remotely resembled the TATAAAA sequence (see Fig. 1A). As further evidence, neither the recombinant hTBP nor the endogenous HeLa TFIID specifically bound to any sequence on the $G_{6} I$ promoter, over a wide protein concentration range that protected an equivalent probe containing the adenovirus major late (AdML) TATA box (data not shown). By this criterion, neither TBP nor any component in the TFIID complex recognized the $\mathrm{G}_{6} \mathrm{I}$ promoter. In the presence of excess TBP or TFIID, nonspecific binding throughout the probe was observed, with the consequence of a high level of random transcription initiating throughout the template (data not shown).

Basal transcription on $\mathrm{G}_{6} \mathrm{I}$ was then assessed. In a partially purified transcription system devoid of Spl, specific initiation was $<0.1 \%$ of the level observed with an equivalent TATA-containing promoter (Fig. 1B, lanes $1,2)$. In the presence of Spl, efficient transcription was restored on $\mathrm{G}_{6} \mathrm{I}$ at the previously defined in vivo start site (Fig. 1B, lane 3; Smale and Baltimore 1989). The $>100$-fold stimulation of transcription observed on $\mathrm{G}_{6} \mathrm{I}$ with the addition of $\mathrm{Spl}$ emphasizes the central role that Spl plays in activating transcription from this TATAless promoter. The failure of $\mathrm{G}_{6} \mathrm{I}$ to support any basal transcription and specifically bind hTBP or the human TFIID (hTFIID) complex relative to its TATA-containing analog leads us to conclude that the $G_{6} I$ promoter represents a bona fide TATA-less template that has no intrinsic ability to recruit TBP or the TFIID complex directly.

\section{Inactivation of TBP}

Having established that the recombinant hTBP and endogenous human hTFIID complex have no intrinsic binding specificity for the $\mathrm{G}_{6} \mathrm{I}$ promoter, the next step was to determine whether TBP played any role in Sp1activated transcription from this promoter. Our previous study revealed that the partially purified HeLa TFIID fraction, which contained at least TBP, the Spl coactivator, and the tethering factor, was required for recon- 
A

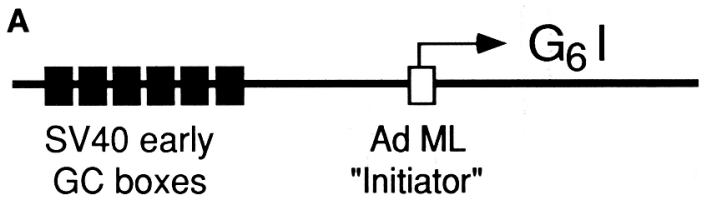

5'-GGCATGCATC TCAATTAGTCAGCAACCATAGTCCCGCCCCTAACTCCGCCCATC CCGCCCCTAACTCCGCCCAGTTCCGCCCATTCTCCGCCCCATGCAGATCTG ATCAGATCTGATCATCGATGAATTCCGTICGTCCTCRTCTCTCTTCCCCTCCTCCATACCC ATCAGACATATATCGATGATTCGCTCG TCCTTCTTCTATATTCCAAATCTATCATCATTCACTCTCATCCCCTCTCCTCAGCCTCAG-3

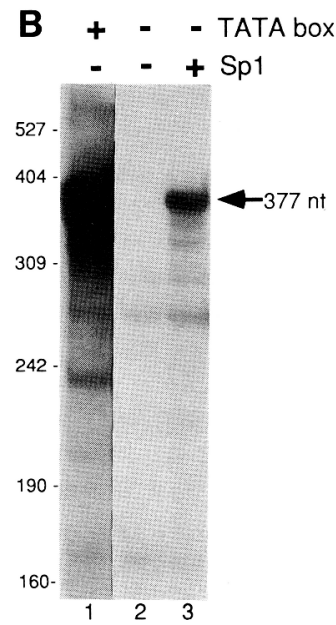

Figure 1. In vitro transcription of a TATA-less promoter. $(A) \mathrm{A}$ schematic and nucleotide sequence of the TATA-less promoter $\mathrm{G}_{6}$ I. The 21-bp repeats (GC boxes) of the SV40 early promoter (solid underline) were placed upstream of the AdML initiator element (broken underline), as described previously (Pugh and Tjian 1990). The transcriptional start site is boxed. (B) Basal and activated transcription on $\mathrm{G}_{6} \mathrm{I}$. HeLa basal initiation fractions (TFIIA, TFIIB, TFIID, and TFIIE/F/polymerase II) were reconstituted on $\mathrm{G}_{6} \mathrm{TI}$ (which is a TATA-containing version of $\mathrm{G}_{6} \mathrm{I}$ ) in the absence of Spl (lane 1), and on $\mathrm{G}_{6} \mathrm{I}$ in the absence (lane 2) or presence (lane 3) of $\mathrm{Sp} 1$ as described previously (Pugh and Tjian 1990). Initiation from the initiator yields a 377-nucleotide transcript. Nucleotide size markers are shown at left.

stituting TATA-less transcription (Pugh and Tiian 1990). Because complete purification of the factors present in this fraction and reconstitution of their activity on $\mathrm{G}_{6} I$ had not been achieved, we were unable to determine unambiguously whether TBP was involved in TATAless transcription. We note that neither the recombinant yeast nor hTBP could replace the endogenous hTFIID fraction for transcription on $\mathrm{G}_{6} \mathrm{I}$, in a system reconstituted with partially purified HeLa basal initiation factors (Pugh and Tjian 1990; data not shown).

A previously described heat treatment protocol (Nakajima et al. 1988), which inactivates TBP in nuclear extracts while leaving the other basal initiation factors and coactivators relatively unaffected, was found to support $\mathrm{Spl-activated} \mathrm{transcription}$ in the presence of added recombinant hTBP on a TATA-containing promoter (Peterson et al. 1990). In contrast, on $\mathrm{G}_{6} I$ we found that the heat-treated nuclear extract was unable to support transcription (Fig. 2, lane 1). Specific initiation was restored by the addition of the partially purified HeLa TFIID (lane 2); surprisingly, however, no amount of recombinant TBP was able to reconstitute transcription on $\mathrm{G}_{6} \mathrm{I}$ (lane 3; data not shown). This suggested that an additional heat-labile factor that copurified with the HeLa TFIID fraction is required for TATA-less transcription. This additional heat-labile activity is likely to be at least part of the previously defined tethering factor, as its activity is dispensable in the presence of a TATA box; that is, as observed previously (Peterson et al. 1990), the recombinant TBP, along with the heat-stable Spl coactivator, restores both basal and high levels of Spl-activated transcription to the heat-treated extract in the presence of a TATA box (Fig. 2, cf. lanes 8,9 with 4,5). Although this experiment provides additional insight into the nature of the tethering factor and further distinguishes it from the coactivator, it does not yield information regarding the requirement of TBP.

In an attempt to specifically inactivate or remove TBP without indiscriminately affecting other important initiation factors, we turned to the use of anti-TBP antibodies. To maximize specificity and minimize any potential nonspecific inhibitory effects on transcription, the polyclonal antibodies were purified by hTBP affinity chromatography. Both the recombinant hTBP and the resulting purified anti-hTBP antibodies were $>95 \%$ pure, as determined by silver-stained SDS-polyacrylamide gels (data not shown). The hTBP antibodies inhibited the production of $\sim 95 \%$ of the RNA transcript initiating from the +1 start site of the TATA-less $\mathrm{G}_{6}$ I template (Fig. 3, lanes 3-8). In contrast, equivalent amounts of affinity-purified

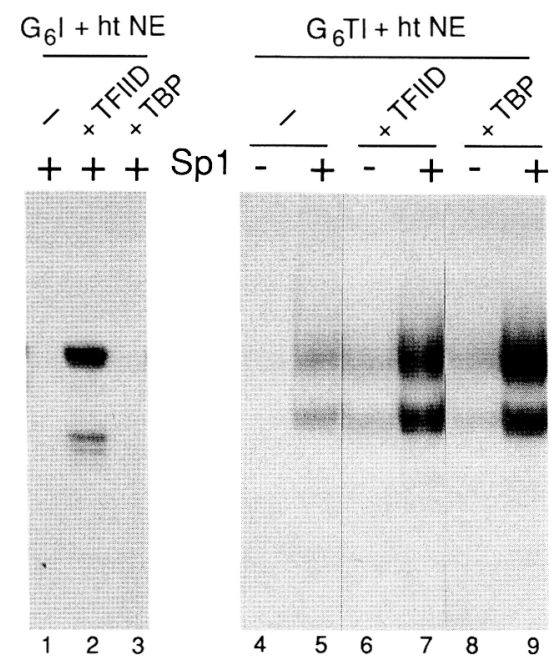

Figure 2. The tethering factor is heat labile. Nuclear extracts were incubated at $47^{\circ} \mathrm{C}$ for $15 \mathrm{~min}$ (Nakajima et al. 1988) and assayed for transcriptional activity on $\mathrm{G}_{6} \mathrm{I}$ in the presence of $\mathrm{SpI}$ (lanes 1-3) or on $\mathrm{G}_{6} \mathrm{TI}$ in the absence or presence of $\mathrm{Spl}$ (lanes 4-9). Lanes 1, 4, and 5 contain no added hTBP; lanes 2, 6, and 7 contain added partially purified HeLa TFIID; lanes 3,8 , and 9 contain $30 \mathrm{ng}$ of hTBP. A broad range of hTBP titrations produced no detectable +1 initiation on $\mathrm{G}_{6} \mathrm{I}$. 

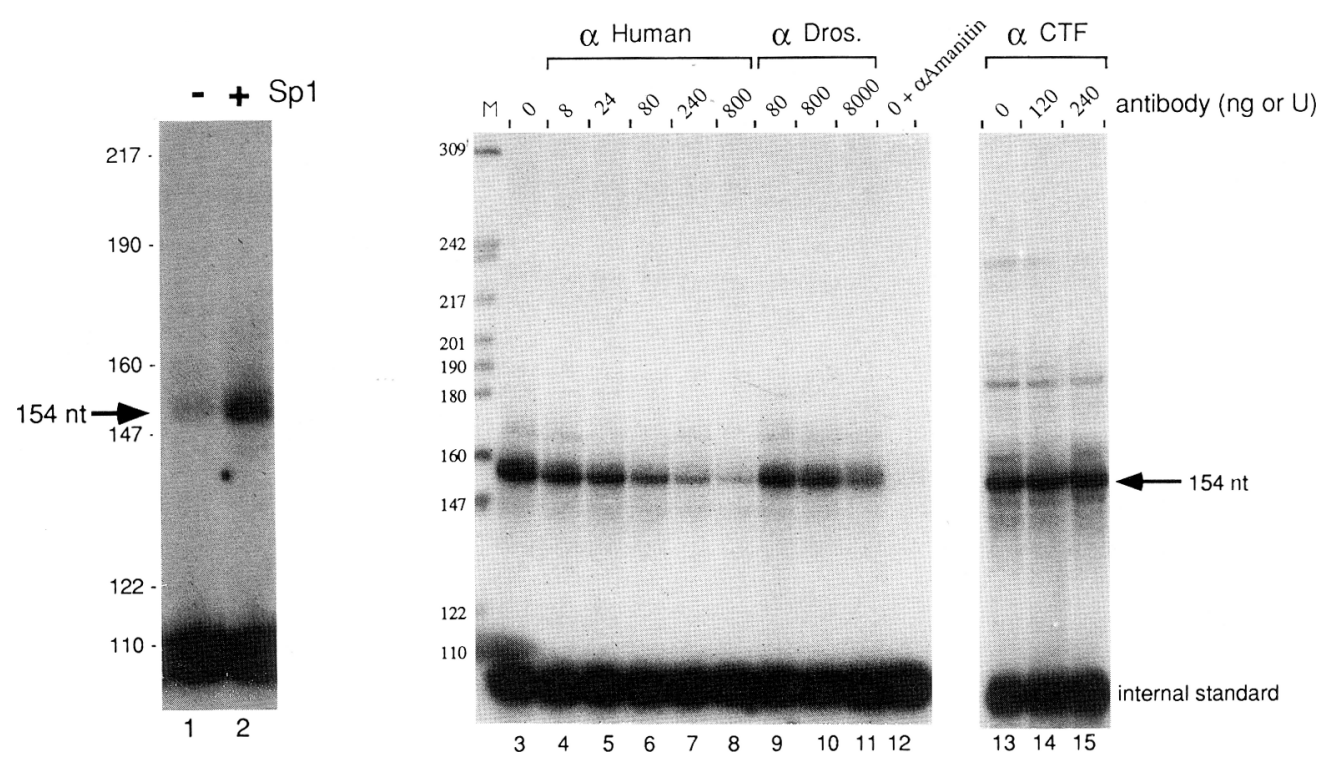

Figure 3. hTBP antibodies specifically inhibit TATA-less transcription. HeLa nuclear extracts $(90 \%$ depleted of Spl $)$ were assayed for transcription on $\mathrm{G}_{6} \mathrm{I}$ in the absence (lane 1) or presence (lane 2) of added Spl by the runoff assay. Although a number of transcription factors present in the HeLa nuclear extract bind to the SV40 21-bp repeats present on $\mathrm{G}_{6} \mathrm{I}$ (Mitchell et al. 1987), lanes 1 and 2 show that $\mathrm{Spl}$ is the dominant activator. Increasing amounts of affinity-purified hTBP antibodies (lanes 3-8), dTBP antibodies (lanes 9-11), or anti-CTF antibodies (lanes 13-15) (as indicated above each lane) were preincubated with the nuclear extract for $2 \mathrm{hr}$ at $4^{\circ} \mathrm{C}$ before assaying the extracts for transcription. Transcripts arising from the +1 start site are 154 nucleotides (nt). The band labeled internal standard arises from an independent reaction involving an internal RNA and $\left[\alpha{ }^{32} \mathrm{P}\right] \mathrm{UTP}$. It serves as a useful internal standard for RNA recovery and comparisons between experiments. The nucleotide molecular mass markers are shown at left. Unit definitions are described in Materials and methods.

control antibodies raised against either the divergent amino-terminal domain of recombinant Drosophila TBP (dTBP antibodies) or the unrelated human transcription factor CTF did not significantly inhibit transcription (Fig. 3, lanes 9-11 and 13-15, respectively). In addition, transcription from $\mathrm{G}_{6} \mathrm{I}$ was completely inhibited by 1 $\mu \mathrm{g} / \mathrm{ml}$ of $\alpha$-amanitin, confirming that transcription was carried out by RNA polymerase II (lane 12). These findings suggested that TBP was involved in Spl-activated TATA-less transcription of the $\mathrm{G}_{6} \mathrm{I}$ template.

\section{Immunodepletion of TBP}

One alternative interpretation of the direct inhibition experiments presented above is that the inhibited factor is not TBP but a different factor that contains one or more cross-reacting epitopes. To identify the proteins that directly bind hTBP antibodies, nuclear extract samples were subjected to Western blot analysis with purified hTBP antibodies (Fig. 4A). The major immunoreactive species migrated with the same molecular mass as the $38-\mathrm{kD}$ recombinant TBP and was therefore likely to be the endogenous TBP (cf. lanes 1 and 3). Two weakly cross-reacting polypeptides of $\sim 65$ and $\sim 90 \mathrm{kD}$ were also present in the nuclear extract. To determine which of the proteins targeted by the antibody resulted in inhibition of TATA-less transcription we performed the following immunodepletion assays. First, purified hTBP antibodies were mixed with the HeLa nuclear extract. Pro- teins bound to the antibodies were then removed by adsorption of the antibody onto protein A-Sepharose resin. When the depleted extract was examined by Western blot, the major depleted species was TBP (Fig. 4A, cf. lanes 2,3 ); the level of the $65-$ and $90-\mathrm{kD}$ proteins remained relatively unaltered. Thus, the antibody appeared to immunodeplete only TBP. The other cross-reacting species might have been abundant low-affinity epitopes that were not depleted efficiently and, therefore, were not likely relevant to transcription initiation.

The next critical test in demonstrating the importance of TBP was to show that immunodepletion of TBP from the extract corresponded to a loss of TATA-less transcription. As shown in Figure 4B (lanes 1-7), increasing amounts of hTBP antibody followed by removal with protein A-Sepharose efficiently depleted the ability of the extract to transcribe $\mathrm{G}_{6} \mathrm{I}$. The control (Drosophila) dTBP antibody had little effect (lane 8).

The specificity of the hTBP antibody was evaluated further by selectively blocking the antibody with purified antigen. Increasing amounts of purified recombinant hTBP or control dTBP were preincubated with the antibody prior to immunodepletion of the extract. The ability of extracts treated with the blocked antibody to support transcription initiating from the +1 start site of $G_{6} I$ was quantitated and shown in Figure 4C. Under these conditions, between 4 and $13 \mathrm{ng}$ of recombinant hTBP was sufficient to neutralize all of the specific antibody in the reaction such that no loss of transcription was ob- 

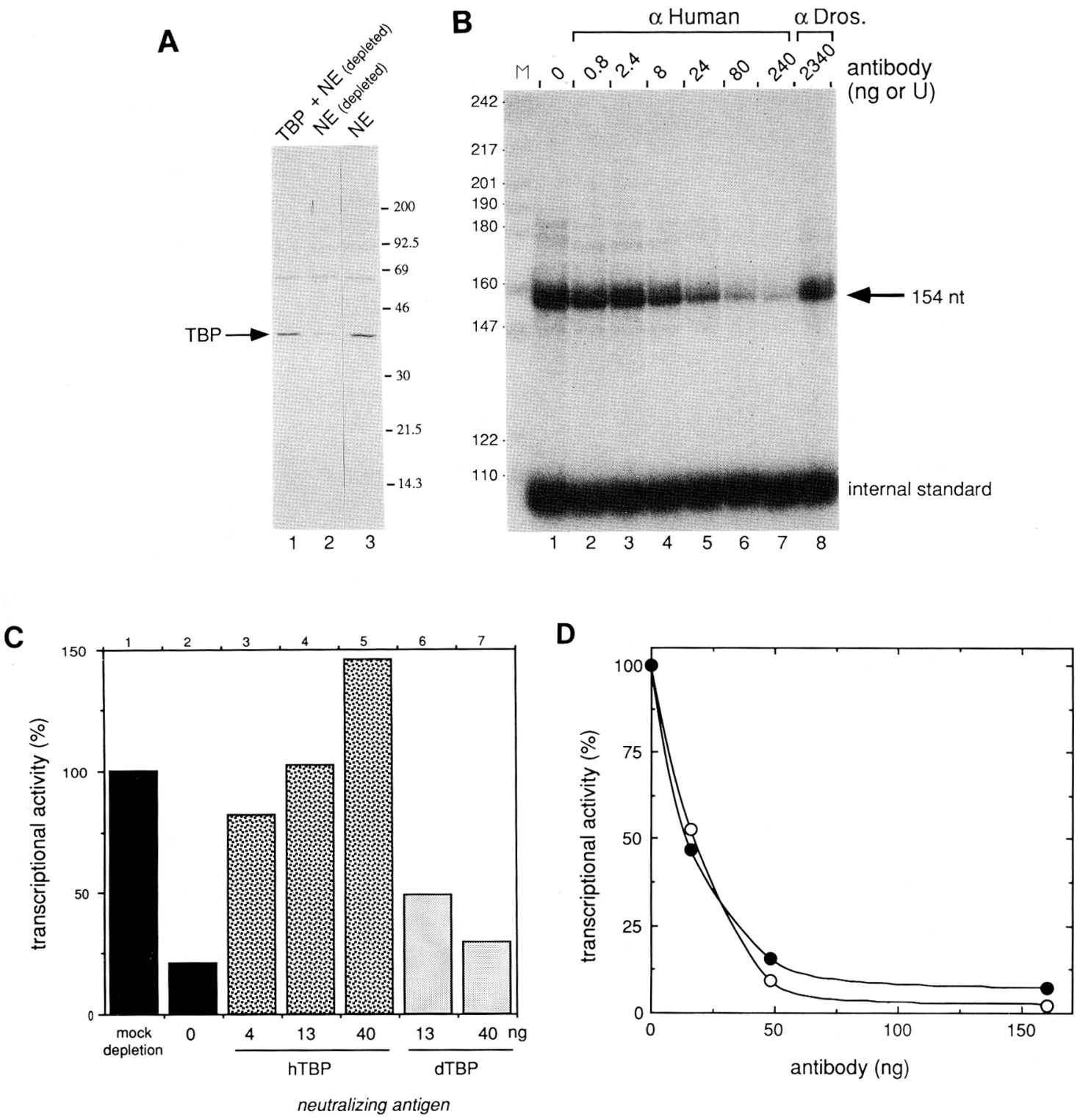

Figure 4. Immunodepletion of the endogenous hTBP. $(A)$ Western blot of TBP. Nuclear extract $(\mathrm{NE} ; 21 \mu \mathrm{g})$, which was either mock-depleted (lane 3) or TBP-depleted (as described below) with $20 \mathrm{ng}$ of hTBP antibody (lane 2), was electrophoresed on an SDS-10\% polyacrylamide gel, transferred onto nitrocellulose, and probed with affinity-purified hTBP antibodies and alkaline phosphataseconjugated goat anti-rabbit $2^{\circ}$ antibody. Lane 1 is the same as lane 2 but also contains $1 \mathrm{ng}$ of purified bacterially expressed hTBP. The endogenous TBP was estimated to be $\sim 0.007 \%$ pure in the nuclear extract. Protein molecular mass $(\mathrm{kD})$ markers are shown at right. (B) Effect of immunodepletion on TATA-less transcription. Increasing amounts of hTBP antibody (lanes 2-7) or dTBP antibody (lane 8) (as indicated above each lane) were incubated with $84 \mu \mathrm{g}$ of nuclear extract for $1 \mathrm{hr}$ at $4^{\circ} \mathrm{C}$. Protein $\mathrm{A}-\mathrm{Sepharose}(2 \mu \mathrm{l}$ hydrated by volume) was then added and mixed intermittently for $1 \mathrm{hr}$ at $4^{\circ} \mathrm{C}$. The mixture was centrifuged at 14,000 rpm (microcentrifuge) for 2 min. The supernatant was assayed for transcription as described in Materials and methods. Identical results were obtained with antibodies derived from two different rabbits. $(C)$ Antibody neutralization. hTBP antibody (80 ng) was preincubated with $0,4,13$, or $40 \mathrm{ng}$ of recombinant hTBP (bars 2-5) or with $0,4,13$, or $40 \mathrm{ng}$ of recombinant dTBP (bars 6-7) for 1 hr at $4^{\circ} \mathrm{C}$. The treated hTBP antibody was then used for immunodepletion of the nuclear extract as described in $B$. The mock reaction contained no antibodies and no recombinant TBP. Treated extracts were assayed for transcription on $\mathrm{G}_{6} \mathrm{I}$ as described in Fig. 3 . Transcripts arising from the +1 start site were quantitated by autoradiography followed by scanning densitometry and are presented in bar graph form. In the experiments where the neutralizing TBP antigen was either in excess or not recognized by the antibody (bars 5-7), a high background of nonspecific transcription was observed throughout the template including near the start site as a result of the high degree of nonspecific binding. This had the effect of artificially increasing the apparent signal of these reactions. $(D)$ Effect of immunodepleting the TFIID fraction on TATA-less transcription and basal TATA-containing transcription. The endogenous TFIID complex was partially purified as described previously (Pugh and Tjian 1990). An aliquot of TFIID (containing $1 \mathrm{ng}$ of TBP as measured by Western blot) was incubated with the indicated amount of hTBP antibody and immunodepleted with protein A-Sepharose as described in $B$. The supernatant was assayed for transcription with the other basal initiation fractions (TFIIA-TFIIF) and Sp1 on $\mathrm{G}_{6} \mathrm{I}$ (O) or for basal TATA-containing transcription in the absence of Spl on $\mathrm{G}_{6} \mathrm{TI}(\mathrm{O})$ as described previously (Pugh and Tjian 1990). Data quantitation is described in C. 
served (cf. bars 3 and 4 with 1 and 2). As a control, dTBP was largely ineffective at blocking the hTBP antibody (bars 6 and 7). These experiments provided additional support that the hTBP antibody was specifically and quantitatively removing the endogenous TBP, thereby rendering the extracts incapable of transcribing $\mathrm{G}_{6} \mathrm{I}$.

In an effort to correlate this apparent TBP dependence of TATA-less transcription with the requirement for TBP at a TATA-containing promoter, Spl-activated transcription on $\mathrm{G}_{6} \mathrm{I}$ was compared side by side to basal (no Spl present) transcription on $\mathrm{G}_{6} \mathrm{TI}$ reconstituted with fractionated basal initiation factors. Increasing amounts of hTBP antibody were preincubated with a partially purified TFIID fraction and were subsequently removed with protein A-Sepharose. The antibody-depleted TFIID fractions were assayed for transcription, and the data were quantitated (Fig. 4D). Increasing amounts of antibody decreased transcriptional activity from both types of promoters with nearly identical profiles, suggesting that TBP was equally important at both types of promoters. Together, the data presented thus far provide compelling evidence that TBP and factors tightly associated with it are essential for transcription at both the TATAless $\mathrm{G}_{6} \mathrm{I}$ and TATA-containing $\mathrm{G}_{6}$ TI promoters.

\section{TBP-associated factors}

The apparent size difference between recombinant TBP and the native TFIID complex suggested that TBP might be only one component of a multisubunit TFIID complex. To address this possibility, the composition of the immunoprecipitated TFIID complex was examined by polyacrylamide gel electrophoresis. Because our starting material was a partially purified TFIID fraction it was first necessary to establish that TBP is the only antigen in this fraction that is capable of being recognized by the anti-hTBP antibodies. Figure 5A shows a Western blot of the TFIID fraction and purified TBP. Only TBP /and minor breakdown products) in the TFIID fraction had avidity for the antibody, thereby establishing it as the only protein in the TFIID fraction likely to be targeted directly by the antibody.

Having established TBP as the sole antibody target in the TFIID fraction, we then examined the immunoprecipitate by SDS-polyacrylamide gel electrophoresis followed by silver staining. Figure $5 \mathrm{~B}$, lane 1 , shows the polypeptide distribution of $<0.3 \%$ of the input TFIID fraction. When the immunoprecipitate was washed extensively and eluted from the protein A-Sepharose with
Figure 5. Purification of TBP-associated factors. $(A)$ Western blot of recombinant bacterial hTBP and the phosphocellulose TFIID fraction. $(B)$ Five micrograms of the partially purified TFIID fraction starting material is shown in lane 1 . It represents $<0.3 \%$ of the starting material immunoprecipitated in lane 12. The TFIID fraction was also immunoprecipitated with anti-hTBP antibodies either free of $(*)$ or blocked (B) with recombinant TBP. The resin was washed and eluted sequentially with a buffer containing $0.2 \mathrm{M} \mathrm{GuHCl}$ (lanes 4,5), $1 \mathrm{M} \mathrm{GuHCl}$ (lanes $6,7)$, and $0.1 \%$ SDS (lanes 8,9 ). The SDS eluted proteins remaining on the resin, such as the antibody and TBP. In lane 9, low levels of a few of the major TAFs also remained. Lane 3 contains $40 \mathrm{ng}$ of purified hTBP (phenyl-5-PW). In lane 12, the immunopurification was scaled up threefold, and the entire purified complex was eluted with $0.1 \%$ SDS. Immunopurified Drosophila TFIID complexes are shown in lane 10 (see also Dynlacht et al. 1991). Molecular mass markers are shown in lanes 2 and 11 . The poor efficiency of the human antibody in immunoprecipitating the endogenous hTBP might indicate that most of the epitopes are covered by the TAFs. $(C)$ Densitometric scan of human TAFs. A gel electrophoresis profile of TAFs eluted with $1 \mathrm{M} \mathrm{GuHCl}$, as described for lane 7 in $B$, is shown below the scan. Apparent molecular masses (in kilodaltons) are shown and were matched only to peaks or fused peaks that corresponded to bands visually identified in at least four independent immunoprecipitation experiments.
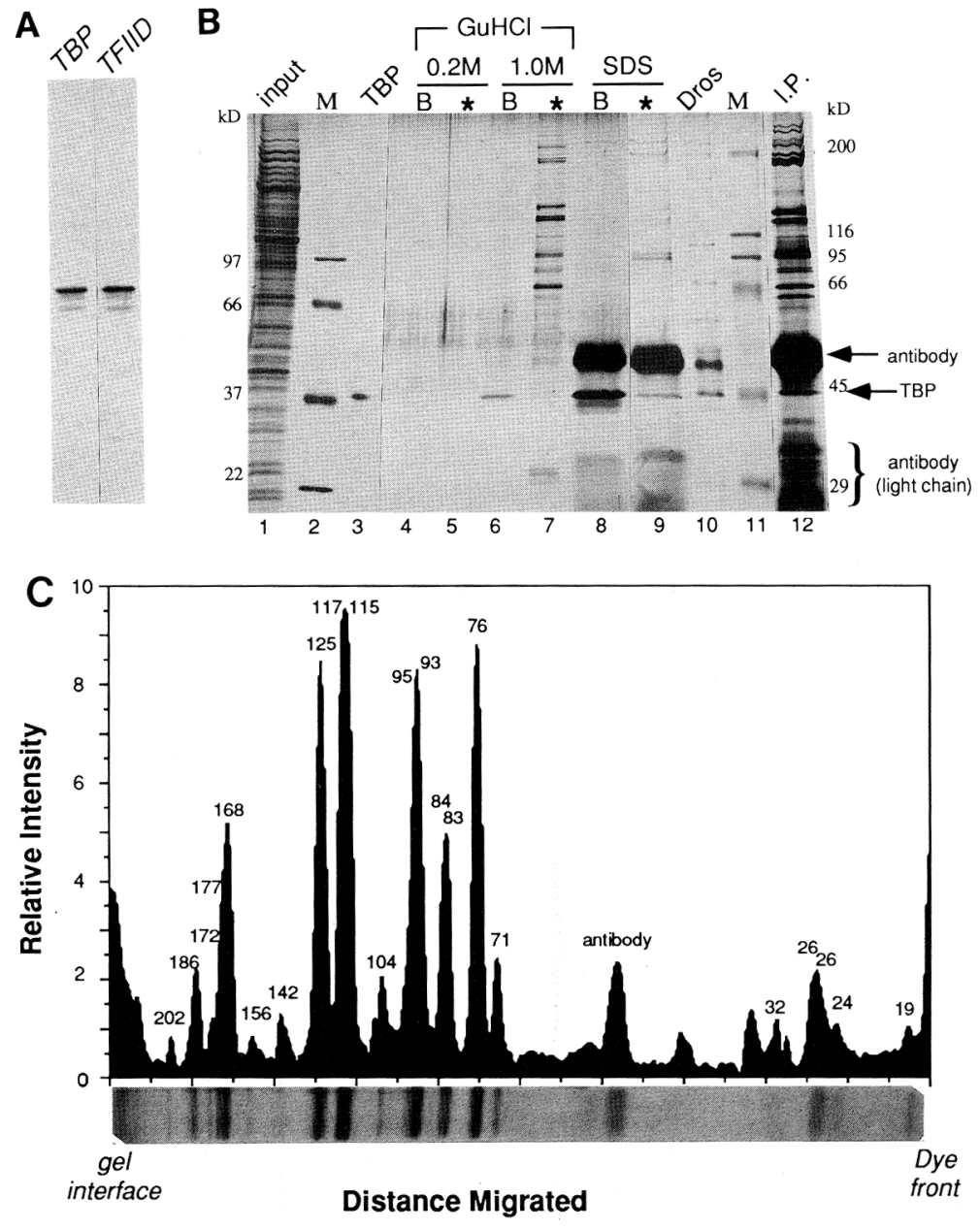
$0.1 \%$ SDS, a large number of proteins were observed to coimmunoprecipitate with the endogenous TBP (Fig. 5B, lane 12). Obviously, many of these proteins could have been nonspecifically adsorbed to the resin, the antibody, or TBP. Therefore, we sought to demonstrate the specificity of these antibodies through a parallel immunoprecipitation in which the antigen-binding sites of the antibody were blocked with recombinant hTBP. Preliminary studies indicated that the tethering factor and coactivator activities were tightly associated with the endogenous TBP and not exchangeable onto exogenous TBP. Therefore, we reasoned that immunoprecipitation carried out with TBP-blocked antibodies would recover few, if any, specific factors and thus would serve as a very stringent control for specificity. In addition, to visualize the various associated factors apart from the large amount of antibody present, we attempted to elute the various subunits from the immunoprecipitated TFIID complex with increasing concentrations of the denaturant guanidine hydrochloride (GuHCl). Parallel studies with Drosophila TFIID suggested that this reagent was effective in stripping TBP-associated factors (TAFs) from dTBP (B.D. Dynlacht and R. Tjian, unpubl.). As shown in Figure 5B, $1 \mathrm{M} \mathrm{GuHCl}$ (lane 7), but not $0.2 \mathrm{M} \mathrm{GuHCl}$ (lane 5), effectively eluted at least 6 major TAFs and at least 10 additional minor TAFs. We estimate a 2000 -fold purification of these TAFs by immunoprecipitation, and $\sim 20,000$-fold enrichment from the crude nuclear extract. In lane 9, the remaining proteins were eluted with $0.1 \%$ SDS. In the control lanes where the antibody was blocked with recombinant $\mathrm{hTBP}$, no major proteins were observed in the $0.2 \mathrm{M} \mathrm{GuHCl}$ (lane 4), $1 \mathrm{M} \mathrm{GuHCl}$ (lane 6), or $0.1 \%$ SDS (lane 8 ) eluate, with the exception of the antibody and recombinant TBP. Thus, the 16 or more TAFs eluted in lane 7 were not contaminants nonspecifically adsorbed to the resin but, instead, appeared to be physically associated with the endogenous TBP. Furthermore, because the antibodies blocked with recombinant hTBP did not become associated with TAFs, the TAFs must be tightly associated with the endogenous TBP and unable to exchange freely.

Molecular weight distribution of the human TAFs (Fig. 5B, lanes 7,12) can be compared to that of the Drosophila TAFs (lane 10) purified in a similar manner. Although there is little correspondence in size of individual TAFs between the two species, a general trend in the pattern of TAF polypeptides is apparent. Thus, TFIID complexes isolated from humans and flies have a qualitatively similar composition and might explain why many human transcription factors function efficiently in Drosophila cells. In Figure 5C, a densitometric scan of the human TAFs is shown along with their apparent molecular masses. Although the relative intensities of the various TAFs reflect the amount of TAFs present, the differences in molecular mass and the potential variable silver staining efficiency of the bands may complicate any quantitative assessment of stoichiometries in the TFIID complex. With that caveat, some TAFs appear to be present in a near stoichiometric ratio while others are clearly substoichiometric. These studies establish that endogenous HeLa TFIID exists as a complex containing a large number of subunits, some of which we suspect to be necessary for tethering factor and coactivator function.

\section{Immunopurified TFIID complexes reconstitute TATA-less transcription}

Parallel studies with the Drosophila TFIID have demonstrated that eluted TAFs can support activator-responsive transcription (coactivator activity; Dynlacht et al. 1991). However, the same TAFs were unable to restore the tethering factor activity on the TATA-less $\mathrm{G}_{6} \mathrm{I}$ promoter (data not shown). This did not come as a surprise because we have found the tethering factor activity to be quite labile, whereas the coactivator activity appears to be more resilient (see Fig. 2D; Pugh and Tjian 1990). Because of these technical limitations we attempted to reconstitute TATA-less transcription with the intact immunopurified TFIID complex attached to the protein A-Sepharose resin. Our rationale was based on two previous observations. First, work by Arias and Dynan (1989) demonstrated that transcription could be reconstituted on a solid support such as Sepharose. Second, close inspection of Figures 3 and $4 \mathrm{~B}$ reveal that the amount of antibody required to immunoprecipitate TFIID was significantly lower than the amount required to directly inhibit activity. Thus, provided excess antibodies are not used to immunoprecipitate TFIID, it should retain a significant amount of transcription activity. The immunoprecipitated TFIID complex was washed extensively to remove any proteins nonspecifically adsorbed onto the resin and added back to a transcription reaction immunodepleted of TFIID activity. As shown in Figure 6, lane 3 vs. lane 1, high levels of transcription were restored on $\mathrm{G}_{6} I$ when the purified immunoprecipitated TFIID complex was added to the reaction. No Spl activation was obtained when protein A-Sepharose alone was added to the reaction, indicating that transcriptional stimulation was specific to the TFIID complex. Not surprisingly, purified recombinant TBP was unable to restore Spl-directed transcription to the TATA-less $\mathrm{G}_{6} \mathrm{I}$ template (lane 2 vs. lane 1) but reconstituted transcription to a TATA-containing promoter (lane 5 vs. lane 4). These results indicate that an intact TFIID complex is essential for transcription at the TATA-less $\mathrm{G}_{6} 1$ promoter. Moreover, TBP appears to be a subunit of this complex and, by itself, is capable of performing some of the activities (i.e., basal transcription) attributed to the TFIID complex but not all of them (i.e., TATA-less transcription).

\section{Discussion}

\section{Promoter assembly via protein-protein interactions}

In an effort to understand how RNA polymerase II can recognize and transcribe the wide variety of promoters that exist in a cell, we have focused on two apparently distinct classes of promoters: TATA-containing and 


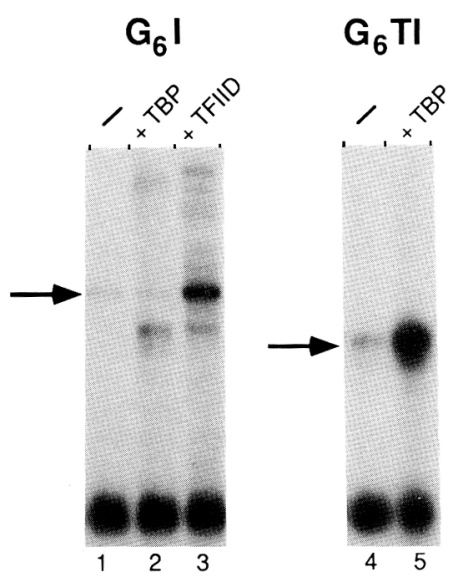

Figure 6. Reconstitution of TATA-less transcription with purified TFIID complexes. Transcription reactions were performed on the $\mathrm{G}_{6} \mathrm{I}$ template (lanes 1-3) and $\mathrm{G}_{6} \mathrm{TI}$ template (lanes 4,5), as described in Materials and methods, by using immunodepleted nuclear extracts supplemented with Spl. In lanes 2 and 5, reactions also contained $20 \mathrm{ng}$ of recombinant hTBP. In lane 3 , immunopurified TFIID complexes containing $20 \mathrm{ng}$ of endogenous TBP were added. The resin containing the immunopurified TFIID complexes (as described in Fig. 5B) was resuspended in buffer G.0 (see Materials and methods) and the indicated amount was assayed.

TATA-less. We suggested previously that the major difference between the two types of templates is the mechanism by which they recruit TFIID to the template. We proposed that TFIID is a complex of many regulatory factors including the TBP (Pugh and Tjian 1990). At TATA-containing promoters, TFIID is recruited directly to the DNA template via sequence-specific interactions between the TATA box and TBP. Variations on this theme might include any DNA element in the promoter that has an intrinsic binding specificity for components of the TFIID complex. In contrast, at true TATA-less promoters that have no intrinsic specificity for TFIID, certain activators such as Sp1, when bound to a GC box, are proposed to recruit the TFIID complex via a tethering factor physically associated with TBP (shown schematically in Fig. 7). Because both types of promoters appeared to require the same basal initiation functions, the bound TFIID complex most likely assembles a similar preinitiation complex at both types of promoters. Promoters that do not bind $\mathrm{Sp} 1$ (or a functionally equivalent factor) but bind other activators might not be able to recruit TFIID efficiently without the help of the TATA box.

Two important assumptions of this tethering model are that (1) TFIID is required at a TATA-less promoter, and (2) TFIID is composed of multiple subunits that include at least the tethering factor and TBP. To address whether TFIID is required at a TATA-less promoter we specifically inhibited TATA-less transcription with affinity purified antibodies directed against TBP. Western blot analysis and immunodepletion studies further confirm that the loss of TATA-less transcription is a result of specific inactivation of TBP by the antibody. Most importantly, immunoprecipitation of TBP revealed that it is physically associated with at least 6 major and 10 minor subunits in addition to TBP. Furthermore, we have reconstituted transcription on the TATA-less $\mathrm{G}_{6} I$ template with the purified TFIID complex. From the data presented in this paper it is reasonable to propose that promoters transcribed by RNA polymerase Il, including those that lack a TATA box, are likely to require one form or another of the TFIID complex for efficient transcription initiation. Recent studies indicate that TBP is also an essential component of RNA polymerase III initiation complexes; therefore, the TBP may serve as a more general eukaryotic transcription initiation factor than believed previously (Margottin et al. 1991; Simmen et al. 1991).

The experiments presented in this paper demonstrate a requirement for a TFIID complex for TATA-less transcription but do not identify the important subunits. Thus, it is formally possible that TBP might not be directly involved. The idea that TBP is present in the complex but not actually participating in the initiation reaction is, however, inconsistent with the finding that TATA-less promoters require both TFIIA and TFIIB (Pugh and Tiian 1990), which directly bind hTBP at a TATA-containing promoter (Buratowski et al. 1989; Peterson et al. 1990). In addition, direct inhibition of TATA-less transcription by TBP antibodies (Fig. 3) argues a functional role for TBP, much as antibody inhibition of the large subunit of RNA polymerase Il implicates its role in transcription (Thompson et al. 1989). If TBP were not part of the initiation process, it would be necessary to propose an ad hoc novel circuitry of interactions for TFIIA and TFIIB that bypasses TBP. Our model provides a simple alternative in which the basic preinitiation complex remains essentially unaltered at

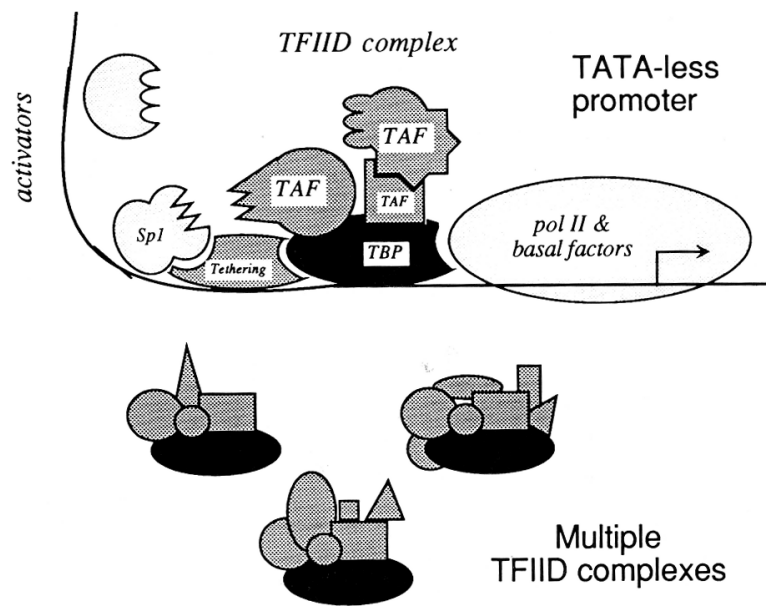

Figure 7. Multiple distinct TFIID complexes. A schematic of factor assembly at a TATA-less promoter involving the TFIID complex. (Below) Schematics of functionally distinct TFIID complexes that might be generated by a specific arrangement of TAFs on TBP (shown in black). Some TAFs might be present in all TFIID complexes. Others might be present in only a subset and are therefore earmarked for certain promoters. 
both TATA-containing and TATA-less promoters but the promoter-binding specificity of the TFIID complex is achieved through protein-protein interactions with its tethering factor and promoter-bound Spl instead of direct protein-DNA interactions between the TBP subunit and the TATA box (Fig. 7). It is likely that TBP can interact directly with the DNA at a TATA-less promoter once it is recruited by protein-protein interactions. Consistent with this proposal, Sp1, to a limited extent, appears to help stably assemble a preinitiation complex at the promoter (B.F. Pugh, unpubl.). In preliminary studies we also looked for recruitment of the TFIID complex to $\mathrm{G}_{6} \mathrm{I}$ in the presence of $\mathrm{Sp} 1$ by DNase I footprint analysis and found that TFIID caused DNase I hypersensitive sites between -30 and -45 , but we were unable to detect significant protection. Whether this reflects the need for additional factors (e.g., TFIIA) to promote stable binding remains to be determined.

The possibility that the TFIID complex gains binding specificity through protein-protein interactions with promoter-bound $\mathrm{Spl}$ has an early precedent in prokaryotic systems. Analogous to a TATA-less promoter, the phage $\lambda p_{\mathrm{RE}}$ promoter lacks a consensus -35 sequences necessary to tether the Escherichia coli RNA polymerase initiation complex to the promoter. As a substitute the $\lambda$ transcriptional regulatory protein $\mathrm{cII}$ binds to its recognition sequence in the -35 region and recruits RNA polymerase presumably through protein-protein interaction (Shimatake and Rosenberg 1981). Substituting the cII protein for the -35 DNA sequence allows $c$ Il to specifically control the initial expression of $c \mathrm{I}$ and thus the decision toward lysogeny or lysis.

\section{Distinguishing TBP, tethering factor, and coactivator}

Although TFIID contains multiple subunits, attributing activities to each has not been straightforward. The following activities have been attributed to the TFIID complex: (1) TATA box-binding factor; (2) basal initiation factor; (3) coactivator; and (4) tethering factor. The first two can be carried out by TBP alone. The coactivator activity is required for sequence-specific activators such as $\mathrm{Sp} 1$ to stimulate the basal level transcription provided by TBP (Peterson et al. 1990; Pugh and Tjian 1990; Dynlacht et al. 1991). Denaturants such as urea and guanidine can be used to separate TBP from coactivators (Dynlacht et al. 1991; Tanese and Tijian 1991; this paper). The tethering factor is required for Spl to activate a promoter that lacks a functional TATA box and is distinct from the coactivator by the following criteria: (1) Mono S chromatography (Pugh and Tjian 1990) or $1 \mathrm{M} \mathrm{GuHCl}$ (B.F. Pugh, unpubl.) destroys tethering activity but not coactivator or TBP activity; and (2) heating a nuclear extract inactivates TBP (Nakajima et al. 1988) and the tethering factor (this paper) but not the $\mathrm{Spl}$ coactivator. These criteria distinguish the tethering factor, coactivator, and TBP activities from one another, but they do not distinguish which TAFs and how many comprise the tethering factor and coactivator activities. Although these two activities might share common subunits, the important point is that all of these activities reside in a multisubunit TFIID "holoenzyme."

\section{Implications of a multisubunit TFIID complex in gene regulation}

The concept of a multisubunit TFIID complex has important ramifications in the regulation and specificity of transcription. TFIID complexes might be heterogeneous in composition (illustrated in Fig. 7), each bearing a distinct combination of TAFs analogous to the combinatorial array of cis-regulatory elements that make each promoter unique. The proper arrangements of TAFs might provide the complementary surface for the unique arrangement of sequence-specific transcriptional regulators at the promoter. Figure 7 shows one possible arrangement for a TATA-less promoter. Each promoter might assemble a more or less distinct regulated initiation complex that can dictate, among other things, the choice of RNA polymerase (I, II, or III) and the overall efficiency of transcription initiation. Although the 16 or more TAFs that comprise the HeLa TFIID complex might seem ungainly at first, we are reminded that a number of macromolecular reactions such as protein synthesis and RNA splicing require a large number of protein and RNA components. Bacteria and yeast have provided useful paradigms for studying higher eukaryotic transcription, but the bacterial $\sigma$-factors and yeast TFIID appear to be monomeric. Transcriptional regulation in yeast might involve coactivators or mediators not tightly associated with TFIID (Berger et al. 1990; Kelleher et al. 1990). A large multisubunit TFIID complex in higher eukaryotic cells might have evolved for the extensive genetic regulation needed by highly specialized cells in a multi-cellular organism.

\section{Materials and methods}

\section{Proteins}

Published procedures were used to prepare the following reagents: HeLa nuclear extracts (Dignam et al. 1983) 90\% depleted of Sp1 (Jackson and Tjian 1989); HeLa basal initiation fractions (TFIIA, TFIIB, TFIID, TFIIE/F/polymerase II) (Pugh and Tjian 1990); and recombinant vaccinia Spl (Jackson and Tiian 1989); recombinant bacterial dTBP (Hoey et al. 1990). hTBP and TFIID purifications are described below. hTBP, dTBP, and CTF peptide rabbit polyclonal antibodies were raised against gel-purified proteins and were antigen/affinity-purified at low $\mathrm{pH}$ (Dynlacht et al. 1991). Purified proteins were quantitated on silver-stained gels and were judged $>95 \%$ pure. Nuclear extracts were quantitated by Bradford, using gamma globulin as a standard.

\section{Transcription}

Transcription reactions shown in Figure 2 contained $84 \mu \mathrm{g}$ of Spl-depleted nuclear extract, $150 \mathrm{ng}$ of Spl (when present), 200 ng of plasmid templates, $4 \mathrm{~mm}$ spermidine, $4 \mathrm{~mm} \mathrm{MgCl}_{2}, 30 \mathrm{~mm}$ $\mathrm{KCl}, 10 \mathrm{~mm}$ HEPES-KOH ( $\mathrm{pH} 7.9$ ), $10 \%$ glycerol, $0.5 \mathrm{~mm}$ DTT, $0.5 \mathrm{~mm}$ ATP, $0.5 \mathrm{~mm} \mathrm{CTP}, 0.5 \mathrm{~mm} \mathrm{GTP}$, and $0.5 \mathrm{~mm}$ UTP in a volume of $20 \mu \mathrm{l}$. Mixtures were preincubated for $30 \mathrm{~min}$ at $30^{\circ} \mathrm{C}$ 
in the absence of NTPs. Reactions were terminated $10 \mathrm{~min}$ after the addition of NTPs, and RNA products were measured by primer extension (Jones et al. 1985).

Transcription reactions shown in Figures 3, 4, and 6 were performed as described above except for the following changes: $0.012 \mathrm{~mm}\left[\alpha^{-32} \mathrm{P}\right] \mathrm{UTP}(5 \mu \mathrm{Ci})$ replaced the $0.5 \mathrm{~mm}$ UTP; $150 \mathrm{ng}$ of poly[d(G-C)] was added; and $20 \mathrm{ng}$ (110 fmoles) of the $\mathrm{G}_{6} \mathrm{I}$ promoter fragment shown in Figure 1A was used as a template. Runoff transcripts were analyzed as described previously (Peterson et al. 1990).

\section{Unit calculations}

This section applies to Figures 3 and 4A. The dTBP antibody had 130-fold more avidity than the hTBP antibody against their respective antigens. To correct for this avidity difference, the amount of dTBP antibody is reported in units, which was calculated by multiplying the amount of antibody (e.g., $60 \mathrm{ng}$ as in lane 11 ) by the relative avidity (130). The hTBP antibody avidity was arbitrarily set at 1 . Relative avidity was determined by Western blots, comparing the amount of antibody needed to generate equal signal intensities from equal amounts of antigen. The anti-CTF antibody is presented in terms of nanograms. The dTBP antibody cross-reacts with hTBP at $1-3 \%$ of the level it reacts with the dTBP and specifically inhibits transcription in a Drosophila nuclear extract (T. Hoey, and R. Tjian, unpubl.).

\section{Recombinant hTBP purification}

pARhTFIID-recombinant $E$. coli BL21 was grown in 12 liters of YT medium, $0.4 \%$ glucose, and $200 \mu \mathrm{g} / \mathrm{ml}$ of ampicillin at $37^{\circ} \mathrm{C}$ to an $\mathrm{OD}_{600}$ of 0.5 . The culture was induced with $0.1 \mathrm{mM}$ IPTG and grown for an additional $60 \mathrm{~min}$ at $30^{\circ} \mathrm{C}$. All subsequent steps were performed at $4^{\circ} \mathrm{C}$. The harvested cells were resuspended in $100 \mathrm{ml}$ of lysis buffer [ $25 \mathrm{mM}$ HEPES at $\mathrm{pH} 7.6,200$ $\mathrm{mM} \mathrm{KCl}, 0.1 \mathrm{~mm}$ EDTA, $12.5 \mathrm{~mm} \mathrm{KCl}, 10 \%$ glycerol, $0.1 \%$ NP-40, $1 \mathrm{~mm}$ DTT, $0.1 \mathrm{~mm}$ phenylmethylsulfonide fluoride (PMSF), $0.1 \mathrm{mM}$ sodium metabisulfite] and incubated with 50 $\mathrm{mg}$ of lysozyme for $15 \mathrm{~min}$. The cells and DNA were disnupted by sonication in two 30 -sec bursts at maximum output. Nucleic acids and bound proteins were precipitated by a slow addition of polymin $P$ to a final concentration of $0.25 \%(\mathrm{vol} / \mathrm{vol})$. The material was stirred for $20 \mathrm{~min}$ and centrifuged for $15 \mathrm{~min}$ at 15,000 $\mathrm{rpm}$ (SS-34 rotor). $\mathrm{KCl}$ was added to the supernatant until its conductivity was equal to that of buffer H.35 (20 mM HEPES$\mathrm{KOH}$ at $\mathrm{pH} 7.9,10 \%$ glycerol, $2 \mathrm{mM} \mathrm{MgCl}, 1 \mathrm{mM}$ DTT, $0.5 \mathrm{mM}$ EDTA, $0.1 \mathrm{mM}$ PMSF, $0.35 \mathrm{M} \mathrm{KCl}$ ) and was then applied to a $40-\mathrm{ml}(2.5 \times 8 \mathrm{~cm})$ column of phosphocellulose equilibrated with buffer H.35. TBP was eluted with buffer $\mathrm{H} 1$ (which contained $1 \mathrm{M} \mathrm{KCl}$ ). The TBP pool was brought to $0.86 \mathrm{M} \mathrm{KCl}$ and $0.5 \mathrm{M}$ ammonium sulfate by the addition of one-sixth volume of buffer H.0 containing $4 \mathrm{M}$ ammonium sulfate. The material was then applied to a $3.7-\mathrm{ml}$ phenyl-5PW high-performance liquid chromatography (HPLC) column equilibrated in the same buffer. The column was washed with buffer H.43 containing $0.25 \mathrm{M}$ ammonium sulfate. The column was developed with a 20-ml linear salt gradient extending down to buffer H.0. TBP eluted at $0.24 \mathrm{M} \mathrm{KCl} / 0.12 \mathrm{M}$ ammonium sulfate and was $>95 \%$ pure. Peak fractions were pooled and stored at $-80^{\circ} \mathrm{C}$, where activity was stable for at least 15 months.

\section{Immunopurification of TFIID}

Nuclear extracts were chromatographed over phosphocellulose in buffer H.1 and washed with buffer H.5, and TFIID activity eluted with buffer H.7. Four micrograms of affinity-purified
hTBP antibodies was added to $400 \mu \mathrm{g}$ of the phosphocellulose TFIID fraction and incubated on ice for $3 \mathrm{hr}$. In the control incubation, the antibody was preincubated for $3 \mathrm{hr}$ on ice with $800 \mathrm{ng}$ of recombinant hTBP purified from $E$. coli, then added to the TFIID fraction. Protein A-Sepharose resin $(20 \mu l$ hydrated bed volume/ was then added to each, and incubation at $4^{\circ} \mathrm{C}$ on a rotating wheel continued overnight. The resin was centrifuged at $2000 \mathrm{rpm}$ (microcentrifuge) for $2 \mathrm{~min}$ at $4^{\circ} \mathrm{C}$. The resin was washed five times, each with $1 \mathrm{ml}$ of buffer H.7. The resin was then sequentially eluted with $60 \mu 1$ of buffer G.0, G.2, G1.0, and, finally, with protein gel loading buffer containing $0.1 \%$ SDS. Buffer $\mathrm{G}$ is the same as buffer $\mathrm{H}$ but also contains $100 \mathrm{~mm}$ potassium glutamate, $0.1 \% \mathrm{NP}-40$, no $\mathrm{KCl}$, and the indicated amount of $\mathrm{GuHCl}$ (e.g., G1.0 denotes $1 \mathrm{M} \mathrm{GuHCl}$ ). Eluted samples were precipitated with trichloroacetic acid, washed with acetone, and loaded onto a $7.5 \%$ polyacrylamide protein gel. After electrophoresis, the gel was silver stained.

\section{Acknowledgments}

We thank G. Peterson for the hTBP antisera and K. Kilomanski for the template constructs. We also thank H. Echols, J. Kadonaga, C. Gross, and the members of the Tjian laboratory for numerous helpful comments on the manuscript. B.F.P is a fellow of the Leukemia Society of America. This work was supported in part by a grant from the National Institutes of Health.

The publication costs of this article were defrayed in part by payment of page charges. This article must therefore be hereby marked "advertisement" in accordance with 18 USC section 1734 solely to indicate this fact.

\section{References}

Arias, J.A. and W.S. Dynan. 1989. Promoter-dependent transcription by RNA polymerase II using immobilized enzyme complexes. J. Biol. Chem. 264: 3223-3229.

Berger, S.L., W.D. Cress, A. Cress, S.J. Triezenberg, and L. Guarente. 1990. Selective inhibition of activated but not basal transcription by the acidic activation domain of VP16: Evidence for transcriptional adaptors. Cell 61: 1199-1208.

Buratowski, S., S. Hahn, L. Guarente, and P.A. Sharp. 1989. Five intermediate complexes in transcription initiation by RNA polymerase II. Cell 56: 549-561.

Carcamo, J., S. Lobos, A. Merino, L. Buckbinder, R. Weinmann, V. Natarajan, and D. Reinberg. 1989. Factors involved in specific transcription by mammalian RNA polymerase II: Role of factors IID and MLTF in transcription from the adenovirus major late and IVa2 promoters. J. Biol. Chem. 264: 7704-7714.

Carcamo, J., E. Maldonado, P. Cortes, M.H. Ahn, I. Ha, Y. Kasai, J. Flint, and D. Reinberg. 1990. A TATA-like sequence located downstream of the transcription initiation site is required for expression of an RNA polymerase II transcribed gene. Genes \& Dev. 4: 1611-1622.

Cavallini, B., I. Faus, H. Matthes, J.M. Chipoulet, B. Winsor, J.M. Egly, and P. Chambon. 1989. Cloning of the gene encoding the yeast protein BTF1Y, which can substitute for the human TATA box-binding factor. Proc. Natl. Acad. Sci. 86: $9803-9807$.

Dignam, J.D., R.M. Lebovitz, and R.G. Roeder. 1983. Accurate transcription initiation by RNA polymerase II in a soluble extract from isolated mammalian nuclei. Nucleic Acids Res. 11: $1475-1489$.

Dynan, W.S. 1986. Promoters for housekeeping genes. Trends Genet. 2: 196-197. 
Dynlacht, B.D., T. Hoey, and R. Tjian 1991. Coactivators associated with the TATA binding protein mediate transcriptional activation in Drosophila. Cell 66: 563-576.

Eisenmann, D.M., C. Dollard, and F. Winston. 1989. SPT15, the gene encoding the yeast TATA binding factor TFIID, is required for normal transcription initiation in vivo. Cell 58: 1183-1191.

Fikes, J.D., D.M. Becker, F. Winston, and L. Guarente. 1990. Striking conservation of TFIID in Schizosaccharomyces pombe and Saccharomyces cerevisiae. Nature 346: 291-294.

Gasch, A., A. Hoffmann, M. Horikoshi, R.G. Roeder, and N.-H. Chua. 1990. Arabidopsis thaliana contains two genes for TFIID. Nature 346: 390-394.

Hahn, S., S. Buratowski, P.A. Sharp, and L. Guarente. 1989a. Isolation of the gene encoding the yeast TATA binding protein TFIID: A gene identical to the SPT15 suppressor of Ty element insertions. Cell 58: 1173-1181.

Hahn, S., S. Buratowski, P.A. Sharp, and L. Guarente. 1989b. Yeast TATA-binding protein TFIID binds to TATA elements with both consensus and nonconsensus DNA sequences. Proc. Natl. Acad. Sci. 86: 5718-5722.

Hoey, T., B.D. Dynlacht, M.G. Peterson, B.F. Pugh, and R. Tjian. 1990. Isolation and Characterization of the Drosophila gene encoding the TATA binding protein, TFIID. Cell 61: 11791186.

Hoffmann, A., M. Horikoshi, C.K. Wang, S. Schroeder, P.A. Weil, and R.G. Roeder. 1990a. Cloning of the Schizosaccharomyces pombe TFIID gene reveals a strong conservation of functional domains present in Saccharomyces cerevisiae TFIID. Genes \& Dev. 4: 1141-1148.

Hoffmann, A., E. Sinn, T. Yamamoto, J. Wang, A. Roy, M. Horikoshi, and R.G. Roeder. 1990b. Highly conserved core domain and unique $\mathrm{N}$ terminus with presumptive regulatory motifs in a human TATA factor (TFID). Nature 346: 387390.

Horikoshi, M., C.K. Wang, H. Fujii, J.A. Cromlish, P.A. Weil, and R.G. Roeder. 1989. Cloning and structure of a yeast gene encoding a general transcription initiation factor TFIID that binds to the TATA box. Nature 341: 299-301.

Horikoshi, M., T. Yamamoto, Y. Ohkuma, P.A. Weil, and R.G. Roeder. 1990. Analysis of structure-function relationships of yeast TATA box binding factor TFIID. Cell 61: 1171-1178.

Jackson, S.P. and R. Tjian. 1989. Purification and analysis of RNA polymerase II transcription factors by using wheat germ agglutinin affinity chromatography. Proc. Natl. Acad. Sci. 86: 1781-1785.

Jones, K.A., K.R. Yamamoto, and R. Tjian. 1985. Two distinct transcription factors bind to the HSV thymidine kinase promoter in vitro. Cell 42: 559-572.

Kambadur, R., V. Culotta, and D. Hamer. 1990. Cloned yeast and mammalian transcription factor TFIID gene products support basal but not activated metallothionein gene transcription. Proc. Natl. Acad. Sci. 87: 9168-9172.

Kao, C.C., P.M. Lieberman, M.C. Schmidt, Q. Zhou, R. Pei, and A.J. Berk. 1990. Cloning of a transcriptionally active human TATA binding factor. Science 248: 1646-1650.

Kelleher, R.J., III, P.M. Flanagan, and R.D. Komherg. 1990. A novel mediator between activator proteins and the RNA polymerase II transcription apparatus. Cell 61: 1209-1215.

Margottin, F., G. Dujardin, M. Gerard, J.-M. Egly, J. Huet, and A. Sentenac. 1991. Participation of the TATA factor in transcription of the yeast $\mathrm{U} 6$ gene by RNA polymerase C. Nature 251: 424-426.

Mitchell, P.J., C. Wang, and R. Tjian. 1987. Positive and negative regulation of transcription in vitro: Enhancer-binding protein AP-2 is inhibited by SV40 T antigen. Cell 50: 847-861.
Muhich, M.L., C.T. Iida, M. Horikoshi, R.G. Roeder, and C.S. Parker. 1990. cDNA clone encoding Drosophila transcription factor TFIID. Proc. Natl. Acad. Sci. 87: 9148-9152.

Nakajima, N., M. Horikoshi, and R.G. Roeder. 1988. Factors involved in specific transcription by mammalian RNA polymerase II: Purification, genetic specificity, and TATA boxpromoter interactions of TFIID. Mol. Cell. Biol. 8: 40284040.

Peterson, M.G., N. Tanese, B.F. Pugh, and R. Tjian. 1990. Functional domains and upstream activation properties of cloned human TATA binding protein. Science 248: 1625-1630.

Pugh, B.F. and R. Tiian. 1990. Mechanism of transcriptional activation by Spl: Evidence for coactivators. Cell 61: 11871197.

Saltzman, A.G. and R. Weinmann. 1989. Promoter specificity and modulation of RNA polymerase II transcription. FASEB J. 3: 1723-1733.

Schmidt, M.C., C.C. Kao, R. Pei, and A.J. Berk. 1989. Yeast TATA-box transcription factor gene. Proc. Natl. Acad. Sci. 86: 7785-7789.

Shimatake, H. and M. Rosenberg. 1981. Purified $\lambda$ regulatory protein cII positively activates promoters for lysogenic development. Nature 292: 128-132.

Simmen, K., J. Bernués, H.D. Parry, H.G. Stunnenberg, A. Berkenstam, B. Cavallini, J.-M. Egly, and I. Mattaj. 1991. TFIID is required for in vitro transcription of the human U6 gene by RNA polymerase II. EMBO I. 10: 1853-1862.

Singer, V.L., C.R. Wobbe, and K. Struhl. 1990. A wide variety of DNA sequences can functionally replace a yeast TATA element for transcriptional activation. Genes \& Dev. 4: 636645.

Smale, S.T. and D. Baltimore. 1989. The "initiator" as a transcription control element. Cell 57: 103-113.

Swick, A.G., M.C. Blake, and J.C. Azizkhan. 1989. Functional analysis of GC element binding and transcription in the hamster dihydrofolate reductase gene promoter. Nucleic Acids Res. 17: 9291-9304.

Tanese, N., B.F. Pugh, and R. Tjian. 1991. Coactivators for a proline-rich activator purified from the multisubunit human TFIID complex. Genes \& Dev. (in press).

Thompson, N.E., T.H. Steinberg, D.B. Aronson, and R.R. Burgess. 1989. Inhibition of in vivo and in vitro transcription by monoclonal antibodies prepared against wheat germ RNA polymerase II that react with the heptapeptide repeat of eukaryotic RNA polymerase II. I. Biol. Chem. 264: 1151111520.

Workman, J.L., I.C.A. Taylor, and R.E. Kingston. 1991. Activation domains of stably bound GAL4 derivatives alleviate repression of promoters by nucleosomes. Cell 64: 533-544. 


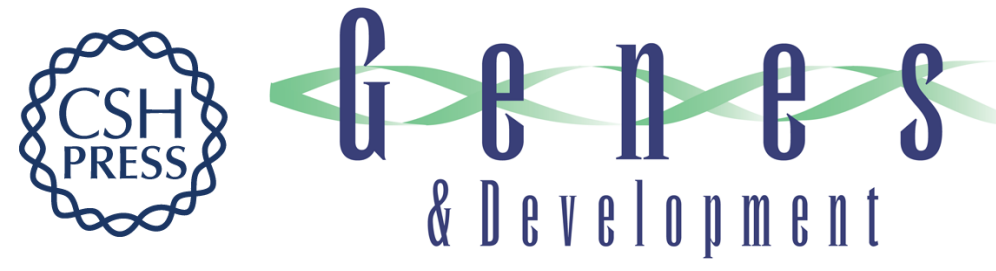

\section{Transcription from a TATA-less promoter requires a multisubunit TFIID complex.}

B F Pugh and R Tjian

Genes Dev. 1991, 5:

Access the most recent version at doi:10.1101/gad.5.11.1935

References This article cites 39 articles, 15 of which can be accessed free at:

http://genesdev.cshlp.org/content/5/11/1935.full.html\#ref-list-1

License

Email Alerting

Service

Receive free email alerts when new articles cite this article - sign up in the box at the top right corner of the article or click here.

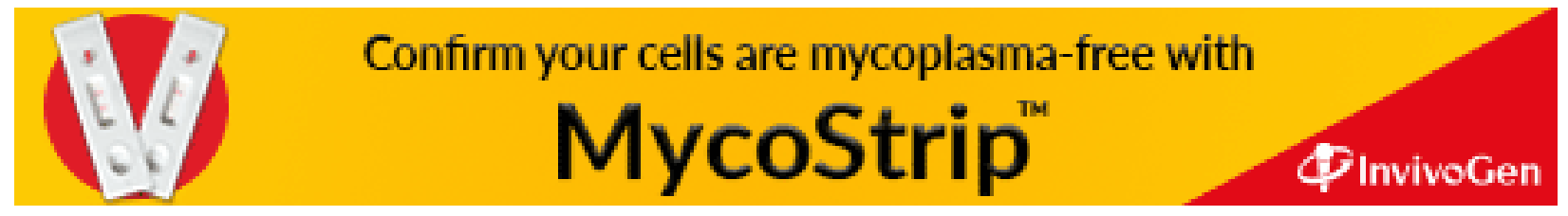

